\title{
Adaptive changes in the relationship between visual and tactual-kinesthetic perception'
}

\author{
JILL RIERDAN AND SEYMOUR WAFNER
}

CLARK UNIVERSITY

Visual and tactual-kinesthetic indications of apparent verticality (adjust a rod so that it appears vertical) were made by $16 \mathrm{Ss}$ under conditions of prolonged exposure to $20^{\circ} \mathrm{clock}$ wise rotation of the visual field. Significant changes were found in the relationship between visual and tactual-kinesthetic verticality. The results are interpreted in terms of a principle of perceptual correspondences.

In contrast to other treatments of perceptual adaptation to visual rearrangement, the present investigation stresses the development of perceptual relationships -here, the relationship between vision and tactualkinesthesis-rather than changes in only one or successive changes in a number of sense modalities (cf., among others, Stratton, 1897; Harris, 1965; Hay \& Pick, 1966). Proceeding from the proposition that perceptual correspondences are disturbed with the introduction of rotating lenses (e.g., in object perception, an object no longer "looks" to be where it "feels" to be), the process of adaptation is presumed to involve the tendency toward the development of new perceptual correspondences.

It is hypothesized that there is a correspondence between visual and tactual-kinesthetic perception of an object before the introduction of rearrangement, i.e., specifically, the angle at which an object is perceived as vertical is the same (minimum angular disparity) both visually and tactual-kinesthetically; that with the introduction of prisms there is a disruption of this perceptual correspondence, i.e., the angle at which an object is seen and felt as vertical is not the same (maximum angular disparity); and that with exposure to visual rearrangement a new perceptual correspondence begins to emerge, i.e., the angular disparity between visual and tactual-kinesthetic adjustments of an object to vertical diminishes.

\section{Method}

Each $\mathrm{S}$ was tested five times for visual and tactualkinesthetic apparent verticality: a pre-test and tests after $0,5,10$, and $15 \mathrm{~min}$. of wearing a monocular prisms system in front of the right eye which produced a $20^{\circ}$ clockwise $(\mathrm{CW})$ rotation of the visual field. During the pre-test the visual field was erect; during the other tests (and between tests) the field was rotated $20^{\circ} \mathrm{CW}$. All tests were conducted in a dark room with $S$ sitting, his head held by a head rest. The visual adjustments always preceded the tactual.

In the visual task, $S$ told $E$ how to change the angle of a luminous rod, $11-1 / 2 \mathrm{in.}$ long and $1 / 8 \mathrm{in}$. wide, and located 75 in. from $S$, so that it looked vertical. Two trials, with initial placement of the rod at either $20^{\circ} \mathrm{CW}$ or $20^{\circ}$ counterclockwise (CCW) of retinal upright, were given on each testing occasion.

In the tactual-kinesthetic adjustment, $S$, with eyes closed, moved a 42 in. long wooden wand up and down the surface of a metal rod-indicator, 24 in. long. With rod-indicator initially placed either $20^{\circ} \mathrm{CW}$ or $20^{\circ}$ $\mathrm{CCW}$ of objective vertical, E, directed by $\mathrm{S}$, changed the angular position of the rod until $S$ reported that it felt vertical as he moved the wand up and down along its surface. Half of the Ss held the wand in their left hand during all tests, half used their right hand.

Five-min. adaptation periods were interpolated between the four tests conducted after introduction of rearrangement. During these periods $S$ viewed the metal rod-indicator and, with the same hand as used in the tactual-kinesthetic task, moved the wooden rod up and down along its surface. S was told to concentrate both on how the rod felt and on how the rod appeared (visually). The metal rod was fixed at an objectively vertical position during these 5-min. periods.

Sixteen Ss, eight males and eight females, were employed.

Results

The angular disparity between the pre-test indications of visual and tactual-kinesthetic verticality, $1.4^{\circ}$, is not significant $(t=1.6, \mathrm{df}=15, \mathrm{p}>.05)$.

As can be seen from Fig. 1, there is a changing

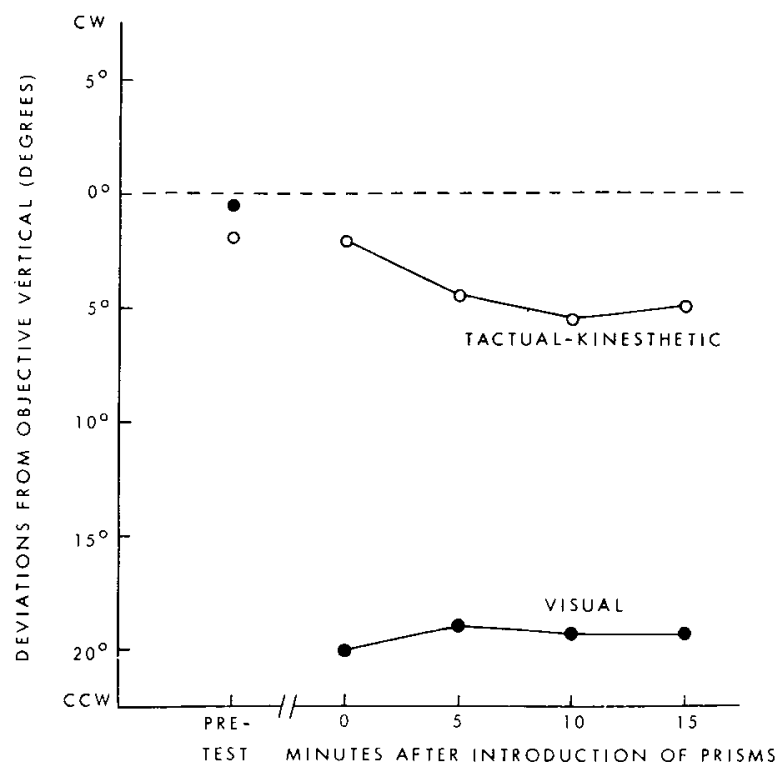

Fig. 1. Changes in the relationship between visual and tactualkinesthetic perception of apparent verticality. 
relationship between visual and tactual-kinesthetic indications of apparent verticality under conditions of prolonged $20^{\circ} \mathrm{CW}$ rotation of the visual field. There is a diminution (most marked between the 0 and 5 min. tests) of the angular disparity between visual and tactual-kinesthetic verticality: the mean differences between these two tasks for the $0,5,10$, and 15 min. tests, respectively, are $18.1^{\circ}, 14.5^{\circ}, 13.7^{\circ}$, and $14.4^{\circ}$. This interaction between "test occasion" and "task" is significant $(F=4.79, \mathrm{df}=3 / 36, \mathrm{p}<.01)$.

\section{Discussion}

The findings may be interpreted as follows: (1) the minimum angular disparity between pre-test indications of visual and tactual-kinesthetic apparent verticality supports the hypothesis that there is, normally, a correspondence between the felt and seen position of an object; (2) the large angular disparity between visual and tactual-kinesthetic verticality in the $0-\mathrm{min}$. test supports the hypothesis that this normal perceptual correspondence is disrupted with the introduction of rearrangement-there is a discrepancy between visual and tactual perception such that an object which feels erect is seen as tilted; and (3) the progressive diminution of angular disparity between visual and tactualkinesthetic apparent verticality is assumed to reflect the tendency toward the emergence of a new perceptual correspondence between these two percepts.

This interpretation is similar to others in two ways. First, in conceptualizing adaptation as the development of perceptual correspondences, it is consonant with Wohlwill's (1966) suggestion that adaptation should be regarded as involving the system of interrelationships linking the channels of vision and proprioception (tactual-kinesthesis). Second, in assuming that awareness of discrepancy (in this experiment Ss received concurrent visual and tactual information about an object, so as to foster the awareness of the discrepancy between vision and tactual-kinesthesis) is a crucial condition for adaptation, this approach resembles that of Howard et al (1965) and Canon (1966).

The developing correspondence of vision and tactualkinesthesis obtained here is largely a function of changes in the latter modality. It may be assumed that the visual percept served as a constant standard which the tactual percept came to approximate. It is premature, however, to conclude that adaptation is a function solely, or even primarily, of change in tactualkinesthesis (proprioception). Although the tactual sense appears more labile than does the visual in this experiment, it may be, as found by Hay \& Pick (1966), that stable visual changes succeed changes in the tactual-kinesthetic (proprioceptive) system. It may also be that greater visual change may occur with adaptation conditions which emphasize the stability of the tactual-kinesthetic percept. This possibility is being explored.

\section{References}

Canon, L. Adaptation to simultaneous displacements of the visual and auditory fields. Paper presented at American Psychological Association Meetings, 1966.

Harris, C. Perceptual adaptation to inverted, reversed, and displaced vision. Psychol. Ret., 1965, 72, 419-444.

Hay, J., \& Pick, H. Visual and proprioceptive adaptation to optical displacement of the visual stimulus. J. exp. Psychol.. 1966, $22,140-142$.

Howard, I., Craske, B., \& Templeton, W. Visuomotor adaptation to discordant exafferent stimulation. J. exp. Psychol., 1965, 70 . 189-191.

Stratton, G. Vision without inversion of the retinal image. Psychol. Rev., 1897, 4, 341-360, 463-481.

Wohlwill, J. Perceptual learning. Annu. Rev. Psychol., 1966, 17. 201-232.

\section{Note}

1. This investigation was supported, in part, by USPHS Grant $M H$ 00348 from the National Institute of Mental Health and, in part, by Grant GY 116, Undergraduate Research Participation Program of the National Science Foundation. 